Research Article

\title{
Wave-Blocking Characteristics of Corrugated Plates under Explosion
}

\author{
Guoliang Yang, ${ }^{1,2}$ Shuai Feng $\mathbb{D}^{1}{ }^{1}$ and Wenjia Huang $\mathbb{D}^{1}$ \\ ${ }^{1}$ School of Mechanics and Civil Engineering, China University of Mining and Technology (Beijing), Beijing 100083, China \\ ${ }^{2}$ State Key Laboratory of Explosion and Science and Technology, Beijing Institute of Technology, Beijing 100081, China
}

Correspondence should be addressed to Shuai Feng; sqt1700602057@student.cumtb.edu.cn

Received 22 June 2019; Revised 29 December 2019; Accepted 22 January 2020; Published 29 February 2020

Academic Editor: Hamid Toopchi-Nezhad

Copyright $($ C 2020 Guoliang Yang et al. This is an open access article distributed under the Creative Commons Attribution License, which permits unrestricted use, distribution, and reproduction in any medium, provided the original work is properly cited.

Corrugated-board explosion-proof wall is the main means to prevent explosion shock wave damage, and it is important to study the effect of different corrugated plates on the shock wave. Using a high-speed schlieren experimental system and an air overpressure test system, the wave-blocking characteristics of different forms of corrugated plates are comprehensively studied. The schlieren images were used to analyze the influence that the corrugation shape of a corrugated plate has on the shock wave propagation characteristics. The results show that the reflection process of the triangular-, trapezoidal-, and half-cylindricalshaped corrugated plates exhibit differences. The number of reflected waves from the triangular corrugated plate is much greater than that from the other corrugated plates, and it will consume more energy. The diffraction wave-front velocity has a trend of initially decreasing and then increasing and is also reduced by different degrees by the reflection. Comparative analysis of the schlieren images and the air shock wave overpressure test shows that plates corrugated with different corrugation shapes decrease the diffraction overpressure peak and exhibit a hysteresis.

\section{Introduction}

Blast explosions cause a huge impact in the production and life of our modern society. The human body, however, has a very limited tolerance for the shock waves emanating from the explosion product. Specifically, an overpressure exceeding just $0.2 \mathrm{~atm}(20 \mathrm{kPa})$ will exert a bad effect on the human body, while few can survive a shock wave exceeding one atmospheric pressure $(101 \mathrm{kPa})$ [1]. Thus, protection from shock waves has always been an important issue. In recent years, scholars at home and abroad have studied shock waves and protection therefrom mainly through model experiments [2,3] and numerical simulations [4-8]. Chen et al. [9] numerically simulated the shock wave field of spherical charge explosion in air and quantitatively studied the power parameters such as peak overpressure, specific impulse, and positive pressure action time of the shock wave. Zhang et al. [10] studied the influence of wall height, proportional explosion distance and explosive position on the overpressure distribution, and fitted the formula for calculating the overpressure after the wall. Nian et al. [11] carried out numerical simulation analysis on the transmission and diffraction effects of flexible explosion-proof walls, compared the variation characteristics of pressure waveforms, and obtained the distribution law of the pressure field behind the wall. Ma et al. [12] and Zong and Bai [13] proposed the design of a specially shaped explosion-proof wall of a new structure and studied the wave-breaking performance and the propagation law of the shock wave by numerical calculation. Yu et al. [14] studied the dynamic response of a trapezoidal corrugated-board explosion wall under blast loading by ANSYS/LS-DYNA finite element simulation software, analyzed the influence of material strain rate effect, and compared the stress distribution and displacement time history curve of different width walls. Shi et al. [15] analyzed the failure mechanisms of three sizes of trapezoidal corrugated-board explosion wall under different explosion loads, obtained a prediction formula for the uniform pressure-impulse (P-I) curve of different explosion-proof walls by fitting, and predicted the antiexplosion 
ability of the trapezoidal corrugated-board explosion-proof wall.

At present, there exists much research on protection from explosive air shock waves. But most of the current research focuses on the process of shock wave transmission, and few scholars have studied the wave-blocking characteristics of the corrugated plate itself. Furthermore, a pressure field comprehensive test system was used to explode experiments on different forms of corrugated steel plates, where visualization of the air shock wave propagation process was obtained with schlieren images. The waveblocking characteristics of different forms of corrugated steel plate for explosion shock waves were studied, and the propagation law of reflection and diffraction was analyzed.

\section{System and Scheme}

2.1. Experimental System. The explosion wave field and pressure field comprehensive experimental test system comprised a high-speed schlieren experimental system and an air shock wave overpressure test system. The comprehensive experimental system is shown in Figure 1. The optical components in the system consisted of a laser, two plane mirrors (plane mirrors 1 and 2), two concave mirrors (concave mirrors 1 and 2), and a beam expander. The light source emitted by the laser became divergent light after passing through the beam expander. The light was then reflected by plane mirror 1 and concave mirror 1 to become parallel light carrying the flow field information. This parallel light was focused on the edge of the blade by a combination of concave mirror 2 and parallel mirror 2. The blade, placed before the high-speed camera, removed some of the focused light that was ultimately captured by the highspeed camera to obtain a schlieren image [16]. The corrugated steel plates were placed horizontally within the parallel laser flow field. The shock wave generated by the shock device impacts the air and changes the air density, whereupon the overpressure was measured via the pressure sensor of the air overpressure test system placed on the back of the steel plate. The high-speed camera located behind the knife edge possessed a shooting speed of 100,000 frames per second. Because a detonating tube was used as the shock device, the explosive equivalent is small and no protective device was needed for the camera.

2.2. Experimental Scheme. Seven types of corrugated steel plates were designed according to the purpose of the experiment, which are shown in Figure 2. There were two types steel plates, transverse (named $F_{\mathrm{S}}, F_{\mathrm{Y}}$, and $F_{\mathrm{T}}$ in Figure 2(a)) and vertically (named $R_{\mathrm{S}}, R_{\mathrm{Y}}, R_{\mathrm{T}}$, and $R_{\mathrm{C}}$ in Figure $2(\mathrm{~b})$ ). The corrugated shapes are trapezoidal (including $F_{\mathrm{T}}$ and $R_{\mathrm{T}}$ ), triangular (including $F_{\mathrm{S}}$ and $R_{\mathrm{S}}$ ), semicylindrical (including $F_{\mathrm{Y}}$ and $R_{\mathrm{Y}}$ ), and noncorrugated (including $R_{\mathrm{C}}$ ). The samples were made by pressing and welding a steel plate $(1.5 \mathrm{~mm}$ thick). These samples were then used for the reflection and diffraction experiments.

In the reflection experiment, because observation of the reflection portion was desired, it was necessary to arrange

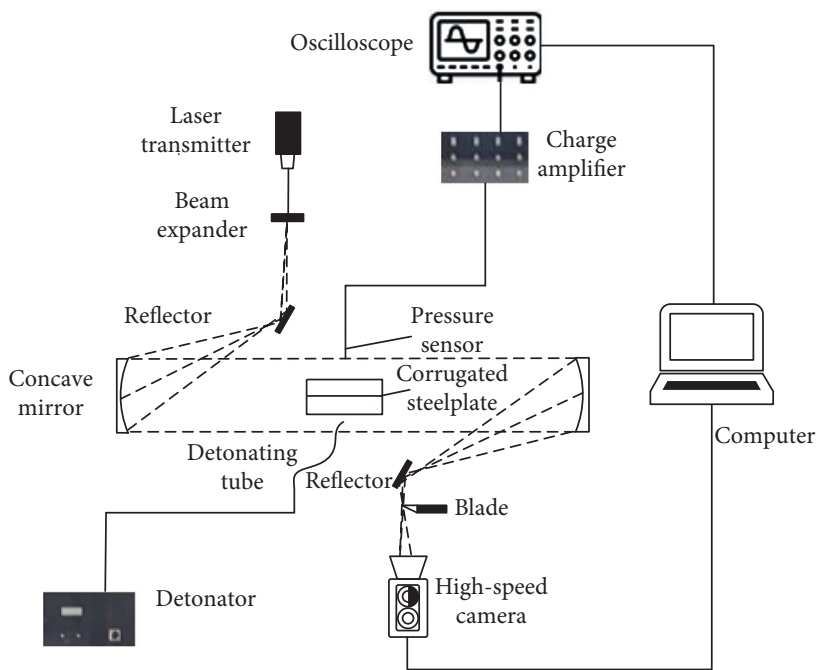

FIGURE 1: Schematic diagram of the comprehensive experimental test system.

lateral corrugations, so that the parallel light could be recorded by the camera. If the corrugations were not arranged laterally for this experiment, the corrugated plate itself would block the parallel light and restrict observation of the reflection process. In addition, the transverse corrugated steel plates were designed to increase the plate area in order to eliminate diffraction interference. The plate sizes with transverse corrugations were $18 \mathrm{~cm}$ long and $18 \mathrm{~cm}$ high.

In the diffraction experiment, the main purpose was to observe the overall diffraction process. Therefore, vertical corrugated steel plates with a height of $5 \mathrm{~cm}$ and a length of $18 \mathrm{~cm}$ were designed with triangular, half-cylindrical, and trapezoidal shapes and no corrugations $\left(R_{\mathrm{S}}, R_{\mathrm{Y}}, R_{\mathrm{T}}\right.$, and $R_{\mathrm{C}}$, respectively, in Figure 2(b)). To ensure that the steel plate was firmly fixed on the horizontal test bench under the action of the shock wave, the lower end of the corrugated steel plate was welded to a footing, which was fixed to the bench.

Because the schlieren observation system has a small observation range, a large-volume explosive was not used for this work. Instead, a detonating tube was used as the shock device initiating the explosion shock wave and the explosive product. In this experiment, a detonating tube with a length of $60 \mathrm{~cm}$ was used as the explosive, with a twisted enameled wire inserted as a detonating probe. The detonator had a power of $2200 \mathrm{~W}$ and was electrically detonated. The shock wave excitation end was placed $3 \mathrm{~cm}$ in front of the center of the steel plate test piece.

When performing the reflection experiment, the test pieces $F_{\mathrm{T}}, F_{\mathrm{S}}$, and $F_{\mathrm{Y}}$ were placed parallel to the optical flow field, so that the high-speed camera could observe the specific reflection process. This arrangement solved the problem of the test piece corrugation itself by blocking the optical path and preventing observation of the actual reflection. When performing the diffraction experiment, the test pieces $R_{\mathrm{S}}, R_{\mathrm{Y}}, R_{\mathrm{T}}$, and $R_{\mathrm{C}}$ were placed as in the reflection experiment. In this way the high-speed camera could obtain 


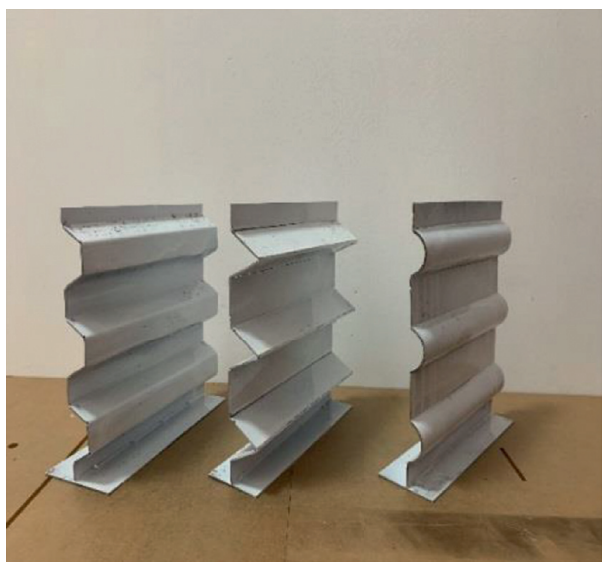

(a)

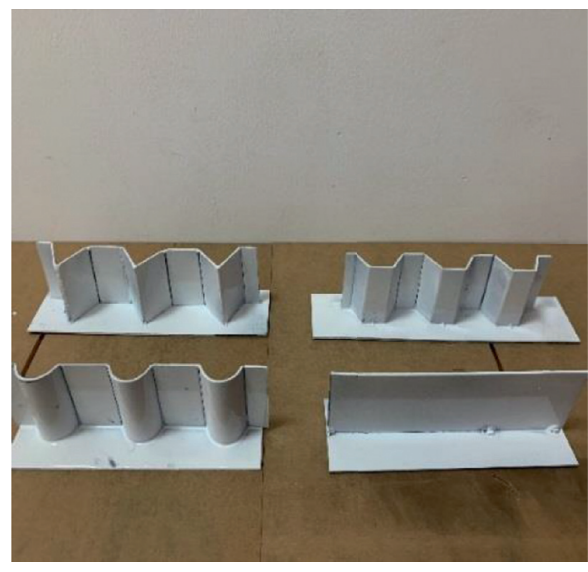

(b)

Figure 2: Corrugated steel plate test pieces used for (a) reflection testing with transverse corrugations shaped as trapezoidal ( $F_{\mathrm{T}}$, left), triangular $\left(F_{\mathrm{S}}\right.$, middle), and half-cylindrical $\left(F_{\mathrm{Y}}\right.$, right) and for $(\mathrm{b})$ diffraction testing with vertical corrugations shaped as triangular $\left(R_{\mathrm{S}}\right.$, upper left), half-cylindrical ( $R_{\mathrm{Y}}$, lower left), trapezoidal $\left(R_{\mathrm{T}}\right.$, upper right), and noncorrugated $\left(R_{\mathrm{C}}\right.$, lower left).

images from the side angle. This arrangement was more intuitive to observe how the shock wave generated by the explosion acted on the steel plate to generate diffraction and enabled recording of the complete diffraction process.

A piezoelectric pressure sensor (CY-YD-202 made by Wuxi Shiao Technology Co., Ltd.) was placed horizontally $5 \mathrm{~cm}$ behind the center of the steel plate and connected to the amplifier and oscilloscope. The trigger voltage was lowered to $20 \mathrm{mV}$, ensuring that a good electrical signal curve could be obtained. The measurable pressure range of the pressure sensor was $0-10 \mathrm{MPa}$, the sensitivity of the charge amplifier was $K=10 \mathrm{mV} / \mathrm{pC}$, and the sensitivity coefficient of the sensor was $\mathrm{Sq}=36.79 \mathrm{pC} / \mathrm{MPa}$.

The number of image frames obtained by the high-speed camera was set by the software, and an image was recorded every $10 \mu$ s.

\section{Results and Analysis}

3.1. Reflection Process of Explosion Shock. During the reflection process, the shock wave follows the law of reflection, where the angle of reflection is equal to the angle of incidence. The different corrugation shapes will change the angle of incidence to form different angles of reflection. Figure 3 shows a comparison of the reflection processes of the tri$\operatorname{angular}\left(F_{\mathrm{S}}\right.$, Figure 3(a)), half-cylindrical $\left(F_{\mathrm{Y}}\right.$, Figure 3(b)), and trapezoidal $\left(F_{\mathrm{T}}\right.$, Figure $\left.3(\mathrm{c})\right)$ corrugated steel plates.

As shown in Figure 3, the initial stage of the reflection process is similar for the three corrugation shapes on the steel sheets. At $t=130 \mu \mathrm{s}$ after detonation, the shock wave is transmitted to the plate surface for all samples, and the triangular corrugated plate produces a reflected wave whose center is the apex of the triangle. The inner side surface of the triangle also causes reflection, and the reflected wave inside the two adjacent corrugations exhibits a symmetric shape. Finally, the reflection from the outer side surface of the triangle bypasses the corrugation and continues to propagate outward. Unlike the triangular corrugated plate, for the half-cylindrical corrugated plate, the wavefront generated by the explosion almost simultaneously reaches the flat surface between the corrugations and the corrugation surface. Upon reflection of the shock wave, the central angle of the reflected wave is larger. The shock wave of the trapezoidal corrugated plate also reaches the plate surface at $t=130 \mu \mathrm{s}$ and forms a larger reflected wave, but the center angle of the reflected wave is moderate. When $t=170 \mu \mathrm{s}$, it can be seen that regular reflection waves appear in front of the different corrugated plates, and the reflected waves on the inner sides of the two adjacent corrugations nearest the explosion gradually approach each other. In addition, the reflected waves from the ground can be observed at this time. Observing the images obtained at $t=260$ and $450 \mu \mathrm{s}$, the reflections produced by the different corrugation shapes began to exhibit differences. As seen for triangular corrugations in Figure 3(a), the shock waves reflected by the inner side of the apex angle continue to propagate and then propagate again to the inner side of the apex angle, whereupon they experience multiple oblique reflections. As shown by $t=260 \mu \mathrm{s}$, at least three reflection ripples are generated in the space between the triangular ripples. The reflection angle of the reflected wave is gradually perpendicular to the inner side, and slowly spreads outward. As seen for half-cylindrical corrugations in Figure 3(b), the shock wave reflected by the half-cylindrical corrugation is again reflected by the half-cylindrical corrugation. The angle of reflection changes gradually and, because the corrugations are half-cylindrical, the repeated reflections are not as numerous as that of the triangular. As shown by $t=260 \mu \mathrm{s}$, there are two reflected waves between the circular ripples. After several reflections, the reflected shock waves from the half-cylindrical corrugation expand outward. As seen for the trapezoidal corrugations in Figure 3(c), the reflection process is more similar to that of the simplified half-cylindrical corrugated plate than that of the triangular corrugated plate. This similarity is owing to the overall similarity and circular reflection. However, owing to the existence of the upper flat base on the trapezoid corrugation shape, there exists a greater chance of vertical reflection that results in fewer reflections 


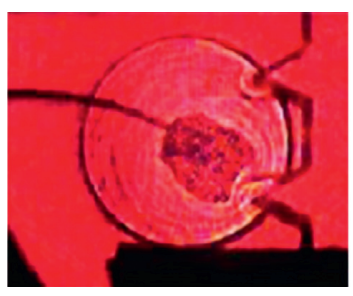

$t=130 \mu \mathrm{s}$

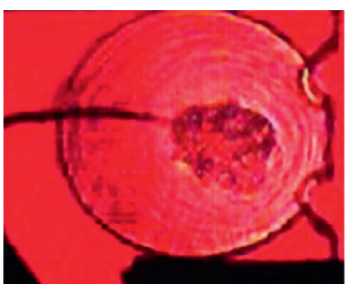

$t=130 \mu \mathrm{s}$

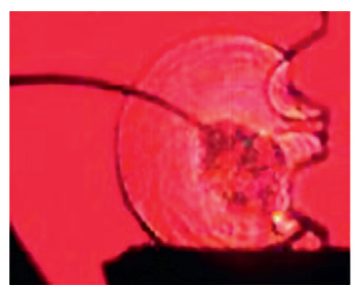

$t=130 \mu \mathrm{s}$

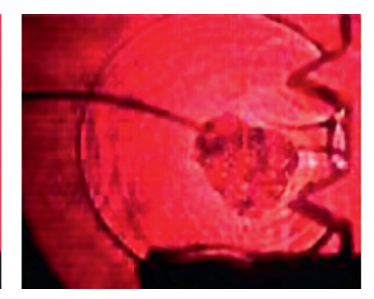

$t=170 \mu \mathrm{s}$

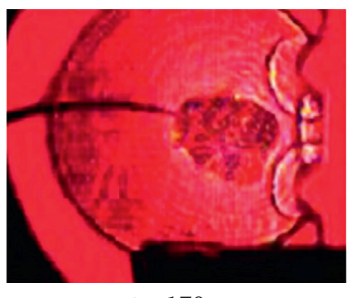

$t=170 \mu \mathrm{s}$

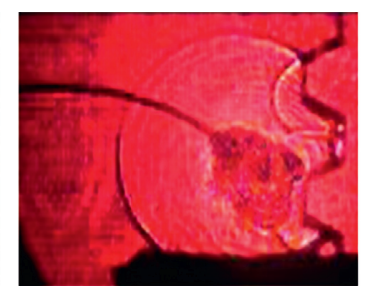

$t=170 \mu \mathrm{s}$

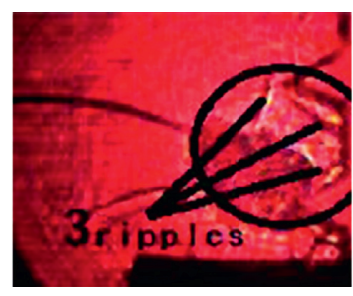

$t=260 \mu \mathrm{s}$

(a)

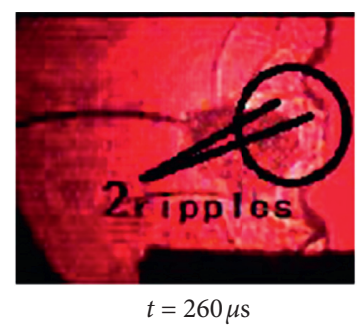

(b)

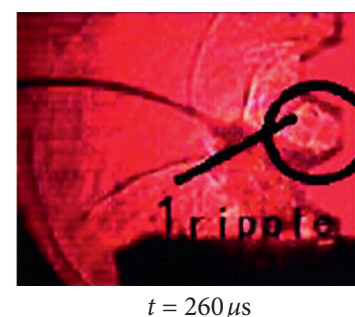

(c)

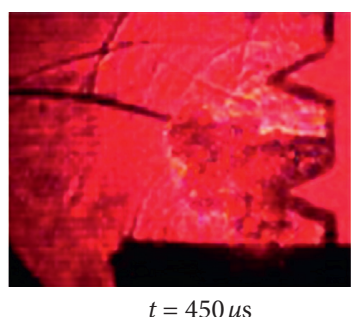

$t=450 \mu \mathrm{s}$
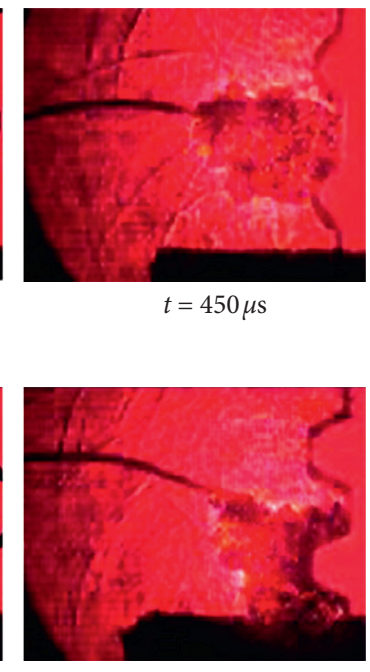

$t=450 \mu \mathrm{s}$

FIGURE 3: Reflection process of the explosion shock wave on the transverse corrugated steel plate with (a) triangular $\left(F_{\mathrm{S}}\right)$, (b) half-cylindrical $\left(F_{\mathrm{Y}}\right)$, and $(\mathrm{c})$ trapezoidal $\left(F_{\mathrm{T}}\right)$ corrugations.

than observed for half-cylindrical corrugations. As shown by $t=260 \mu \mathrm{s}$, there is only one reflected wave between trapezoidal ripples.

In general, the reflection of shock waves exhibited different reflection effects from the differently shaped corrugated steel sheets. Owing to the shape of the triangular corrugated steel plate, increasingly complex reflection processes will emerge between the two adjacent corrugations, which will consume the energy of the shock wave and delay its propagation. The half-cylindrical corrugated steel sheets will form more reflections because of the larger reflection angle, but the small corrugation height restricts the formation of complex multiple reflections. The trapezoidal corrugated steel plate has a flat-topped corrugation similar to a noncorrugated steel plate, which is more prone to ordinary regular reflections. However, because of the existence of the angled sides of the trapezoid, complex reflections between two adjacent corrugations will occur to some extent. The complex reflection process between these adjacent trapezoidal corrugations consumes the energy of the incident shock wave and increases the reflection process time, causing hysteresis of the diffraction.

3.2. Diffraction Process of Explosion Shock. Figure 4 shows the diffraction process of the explosion shock wave acting on the noncorrugated $\left(R_{\mathrm{C}}\right.$, Figure $\left.4(\mathrm{a})\right)$, triangular $\left(R_{\mathrm{S}}\right.$, Figure $4(\mathrm{~b}))$, half-cylindrical $\left(R_{\mathrm{Y}}\right.$, Figure $\left.4(\mathrm{c})\right)$, and trapezoidal $\left(R_{\mathrm{T}}\right.$, Figure $\left.4(\mathrm{~d})\right)$ steel plates. The overall diffraction propagation process for the four different corrugation shapes is similar, but the specific details are different. For each type of corrugated steel plate, we obtained a specific schlieren image at four time points $(60,160,220$, and $320 \mu$ s) for observation.

The observations in Figure 4 show that the different shapes of the corrugated steel plates induce different propagation states upon the diffraction shock wave generated by the explosion. Analysis of the schlieren images at $t=160$ and $220 \mu$ s after detonation shows that the diffraction wavefront of the triangular steel plate (Figure 4(b)) is smaller than that of the other corrugated shapes, and the wavefront transmits for smaller distances. This is because the triangular steel plate forms a more complex reflection process for incident overpressure, increasing the reflection time and delaying the vertical diffraction propagation. Different corrugation shapes have different effects on the diffraction. Table 1 shows the distances of the diffracted shock waves from different corrugated plates at $160 \mu$ s and $220 \mu \mathrm{s}$. From Table 1, we know the wavefront propagation distances for the different metal sheets are ordered as $R_{\mathrm{S}}<R_{\mathrm{Y}}<R_{\mathrm{T}}<R_{\mathrm{C}}$. This result indicates that the triangular corrugated plate exhibits the most delayed diffraction, followed by 


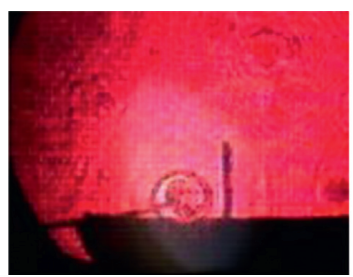

$t=60 \mu \mathrm{s}$

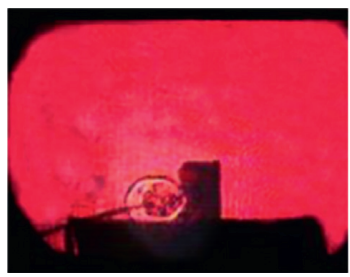

$t=60 \mu \mathrm{s}$

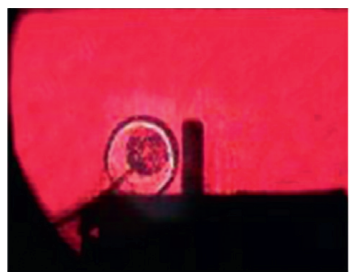

$t=60 \mu \mathrm{s}$

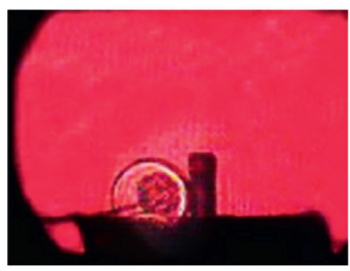

$t=60 \mu \mathrm{s}$

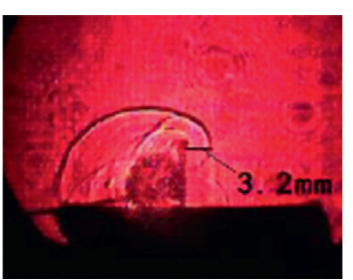

$t=160 \mu \mathrm{s}$

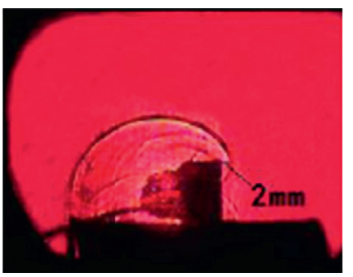

$t=160 \mu \mathrm{s}$

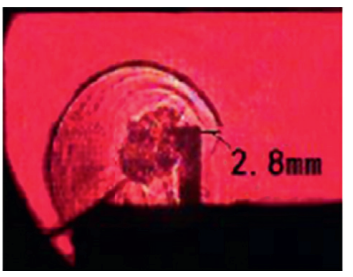

$t=160 \mu \mathrm{s}$

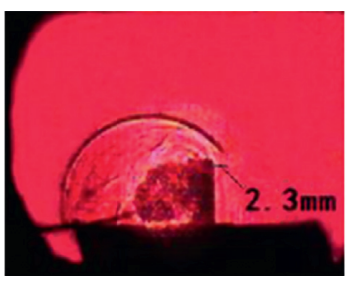

$t=160 \mu \mathrm{s}$

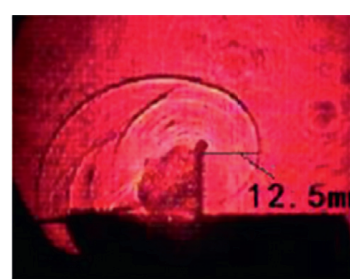

$t=220 \mu \mathrm{s}$

(a)

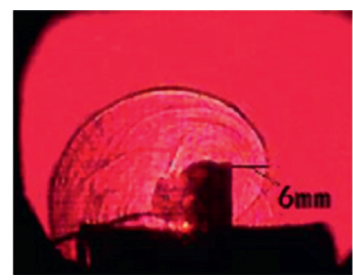

$t=220 \mu \mathrm{s}$

(b)

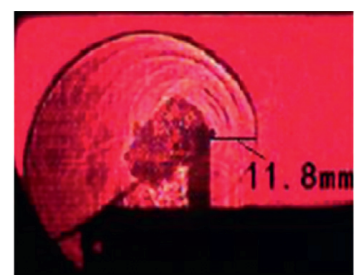

$t=220 \mu \mathrm{s}$

(c)

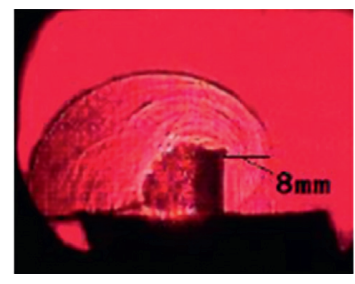

$t=220 \mu \mathrm{s}$

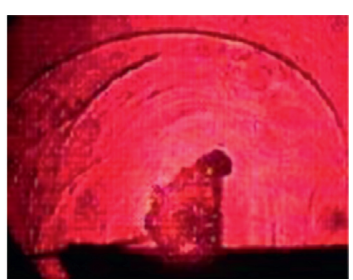

$t=320 \mu \mathrm{s}$

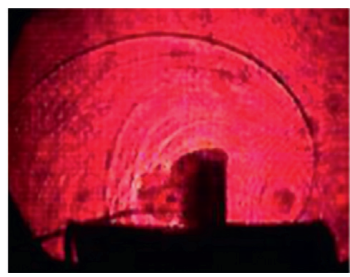

$t=320 \mu \mathrm{s}$

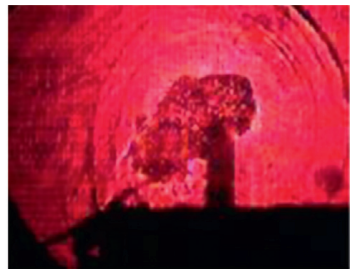

$t=320 \mu \mathrm{s}$

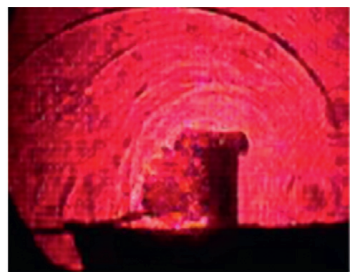

$t=320 \mu \mathrm{s}$

(d)

FIGURE 4: Diffraction process of the explosion shock wave on the vertical corrugated steel plate with (a) no corrugations $\left(R_{\mathrm{C}}\right)$, (b) triangular $\left(R_{\mathrm{S}}\right)$, (c) half-cylindrical $\left(R_{\mathrm{Y}}\right)$, and $(\mathrm{d})$ trapezoidal $\left(R_{\mathrm{T}}\right)$ corrugations.

TABLE 1: Distance between diffraction wave and corrugated plate.

\begin{tabular}{lcccc}
\hline & No corrugations $\left(R_{\mathrm{C}}\right)$ & Triangular $\left(R_{\mathrm{S}}\right)$ & Half-cylindrical $\left(R_{\mathrm{Y}}\right)$ & Trapezoidal $\left(R_{\mathrm{T}}\right)$ \\
\hline$t=160 \mu \mathrm{s}$ & $3.2 \mathrm{~mm}$ & $2 \mathrm{~mm}$ & $2.8 \mathrm{~mm}$ & $2.3 \mathrm{~mm}$ \\
$t=220 \mu \mathrm{s}$ & $12.5 \mathrm{~mm}$ & $6 \mathrm{~mm}$ & $11.8 \mathrm{~mm}$ & $8 \mathrm{~mm}$ \\
\hline
\end{tabular}

half-cylindrical corrugated plates, trapezoidal corrugated plates, and, finally, noncorrugated plates.

In addition, it can be seen in Figure 4(a) that in addition to the reflected shock waves from the ground and from the original steel plate and the newly formed diffraction shock wave, many other shock waves are present in the circle. This is because the shock wave generated by the explosion is not a single shock wave but is composed of a group of shock waves. Thus, there are many outwardly diffusing wavefronts in the outermost shock wave that can be observed. When the shock wave is diffracted, it gradually spreads backward to the corrugated steel plate. When the shock wave reaches the horizontal plane, it interacts with the ground to form a Mach shock wave that gradually propagates to the back of the corrugated steel plate.

3.3. Wave Velocity. To study the propagation characteristics of diffraction shock waves from the different steel plates, the diffraction wave velocity was further analyzed and the diffraction law of the explosion shock wave was compared for the different steel plates.

To extract the shock wave-frontal velocity, Yang et al. proposed that the leading edge of the shock wavefront is 
smooth, thus making it easy to obtain the displacement and speed of the shock wave [16]. The impact of the explosive production on the shock wave transmission is relatively small and therefore was not studied here. For each of the differently shaped corrugation steel plates, the schlieren images after the diffraction process were analyzed, where the interval between each adjacent image was $\Delta t=10 \mu \mathrm{s}$. The recording was started with the top and back of the steel plate chosen as the coordinate origin. The horizontal positions of a specific diffraction wavefront in two adjacent pictures are labeled $x_{1}$ and $x_{2}$, and thus the horizontal displacement difference is $\Delta X=x_{2}-x_{1}$ and the horizontal velocity of the wavefront is $V=\Delta X / \Delta t$. Using these calculated values, the diffraction shock wave velocity and displacement versus time is plotted (Figure 5).

From Figure 5, we can determine how the wave velocity and displacement changes over time. When analyzing the wavefronts from the different steel plates at the same time after detonation, the position of the wavefront propagation is different. The displacement of the wavefront from the triangular $\left(R_{\mathrm{S}}\right)$ steel plate is always the smallest, followed in increasing displacement by the half-cylindrical $\left(R_{\mathrm{Y}}\right)$, trapezoidal $\left(R_{\mathrm{T}}\right)$, and noncorrugated $\left(R_{\mathrm{C}}\right)$ steel plates. This indicates that the diffraction shock wave generated by the explosion will have delayed diffraction on different corrugated plates. The diffraction shock wave transmission of the triangular $\left(R_{\mathrm{S}}\right)$ steel plate is the most obvious, which is consistent with the results of the diffraction process (Section 3.2).

Observing the wavefront velocity curve in Figure 5, it is seen that the wave velocity of the diffraction propagation for steel plates with different-shaped corrugations is basically regular. The law is that, after the diffraction occurs, the wave velocity of the shock wave will initially decrease and then exhibit an increasing trend. This behavior is because, as the shock wave is diffracted, the propagation distance increases and the energy is slowly consumed and the propagation speed is reduced. However, at about $250 \mu \mathrm{s}$, the transmitted diffraction shock wave and the ground action produce a Mach reflection wave that interacts with the original diffraction shock wave to gradually increase the wavefront propagation speed. At the same time, the order of the different steel plates for initial diffraction speed is $R_{\mathrm{S}}<R_{\mathrm{T}}<R_{\mathrm{Y}}<R_{\mathrm{C}}$, which indicates that the corrugated steel plates of different shapes have different energy consumptions for shock waves compared with the noncorrugated steel plate. The triangular corrugated steel plate has the highest shock wave energy consumption, so that the wave velocity transmitted by the diffraction is also small. This result is also consistent with the experimental phenomenon obtained by the reflection experiment.

3.4. Wave Overpressure. The original load signal was obtained from the oscilloscope, and the valid segment needed to obtain the required basic charge signal data was selected and substituted into the formula [17], whereby the unprocessed overpressure signal data were obtained by calculation. Normalization and filter smoothing processes were performed on the Origin software to eliminate interference factors such as noise. The diffraction overpressure time

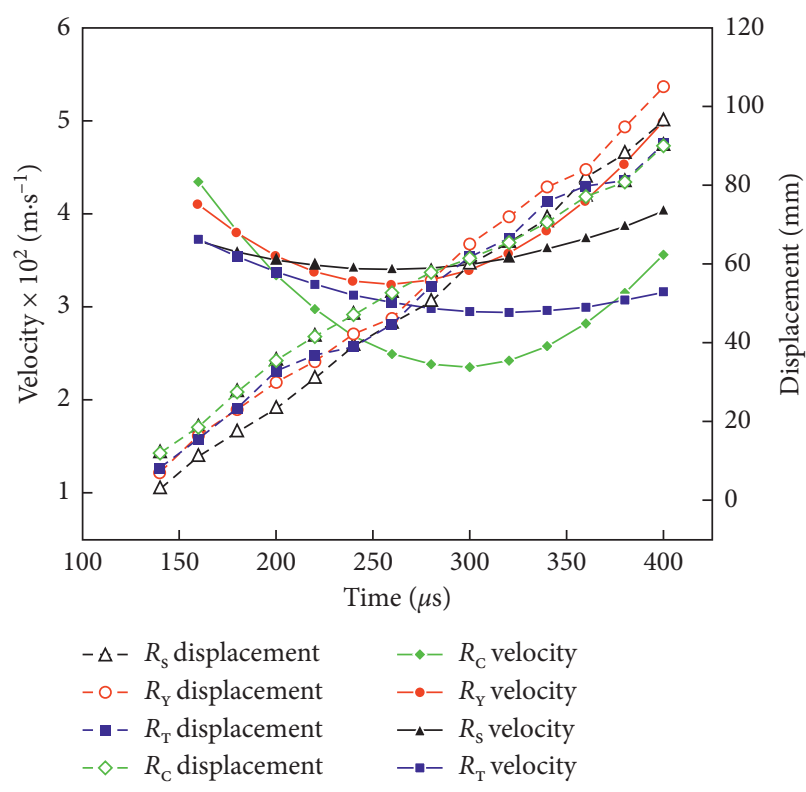

FIGURE 5: Explosion diffraction shock wave displacement velocity versus time curve.

history curve at $5 \mathrm{~cm}$ behind the steel plates with four different shapes was thus obtained. Because the actual experimental specimens were small in size and the signal dose was extremely small, the overpressure value was relatively small. However, the overpressure results are basically in line with the estimated situation. The diffraction overpressure vs. time for the corrugated steel plates with different shapes is plotted in Figure 6.

It can be seen from Figure 6 that the diffraction overpressure propagation process for the different steel plates is roughly the same at equivalent points of the explosion. All steel plates initially experience a positive pressure and then a negative pressure and ultimately tend to a balance. This result is consistent with the diffraction process seen from the schlieren images and is consistent with the law of shock wave propagation. The diffraction overpressure presents two distinct positive phase peaks initially, and the absolute value of the negative phase is smaller than the absolute value of the positive phase. These two results are consistent with the conclusions of $\mathrm{Mu}$ and Wang [4].

As the diffraction overpressure propagates to the sensor, the sensor measures two distinct positive pressure peaks. The first peak is the diffraction of the overpressure shock wave, and the second is the overpressure reflected by the horizontal working surface. Shao and Zhao [5] also previously observed this phenomenon.

Further analyzing Figure 6, it is found that the time of arrival of the shock wave overpressure and the peak of the positive and negative overpressure differ significantly for the different steel plates. The peak time of the post-plate diffraction overpressure of the $R_{\mathrm{C}^{-}}, R_{\mathrm{T}^{-}}, R_{\mathrm{Y}^{-}}$, and $R_{\mathrm{S}^{-}}$steel plates are $175,198,186$, and $216 \mu \mathrm{s}$, respectively. Among the corrugated steel plates, the peak of the diffraction overpressure of the RS-steel plate comes at the latest. This is consistent with the experimental phenomena seen in 


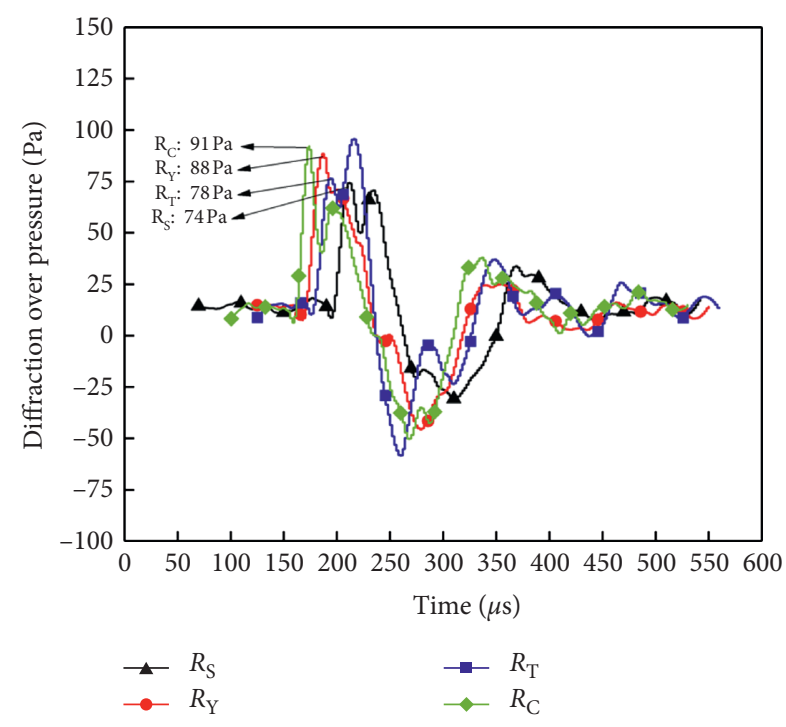

FIGURE 6: Diffraction overpressure as a function of time for the various corrugation shapes on steel plates.

Figure 4, and it is clear that the diffraction overpressure of the triangular steel plate lags behind that of the other steel plates, where the order of diffraction overpressure arrival time is $R_{\mathrm{C}}, R_{\mathrm{Y}}, R_{\mathrm{T}}$, and $R_{\mathrm{S}}$.

Furthermore, the peak value of the diffraction overpressure for the $R_{\mathrm{S}^{-}}, R_{\mathrm{T}^{-}}, R_{\mathrm{Y}^{-}}$, and $R_{\mathrm{C}^{-}}$steel plates are 74,78 , 88 , and $91 \mathrm{~Pa}$, respectively. It is evident that the different shapes of the corrugated steel plates have a great influence on the diffraction overpressure.

\section{Conclusions}

(1) The shock wave reflection process follows the law of reflection, and different corrugation shapes can affect the reflection process and consume more energy. Triangular corrugation reflections are more complex than other corrugated shapes.

(2) The diffraction wavefront velocity has a law of initially decreasing and then increasing and is also affected by reflection. The order of the different steel plates for wavefront velocity is $R_{\mathrm{S}}<R_{\mathrm{T}}<R_{\mathrm{Y}}<R_{\mathrm{C}}$.

(3) Compared with the vertical steel plate, the shock wave diffraction of the different corrugated steel sheets produce different degrees of hysteresis, and the peak overpressure is correspondingly reduced. It is not difficult to see that different corrugated steel plates do have a great influence on diffraction overpressure, and the reduction effect of diffraction overpressure is as follows: $R_{\mathrm{S}}$-steel plate $>R_{\mathrm{T}}$ steel plate $>R_{\mathrm{Y}}$ steel plate $>R_{\mathrm{C}^{-}}$steel plate. The experimental phenomenon of triangular corrugated steel plate is particularly obvious.

\section{Data Availability}

The data used to support the findings of this study are available from the corresponding author upon request.

\section{Conflicts of Interest}

The authors declare no conflicts of interest.

\section{Authors' Contributions}

G. Y. and S. F. conceived and designed the experiments; J. Z. performed the experiments; S. F. and W. H. analyzed the data; S. F. wrote the paper.

\section{Acknowledgments}

This paper was supported by the opening project of the State Key Laboratory of Explosion Science and Technology (Beijing Institute of Technology). The opening project number was KFJJ19-10M.

\section{References}

[1] Li Bingcang and Yang Zhihuan, "Application of Explosive Weapons in Modern Warfare and Its Injury Mechanism," Medical Information of the Chinese People's Liberation Army, vol. 5, pp. 251-253, 1996.

[2] Q. Zhang, Y. Zhang, and X. Nian, "Effect of concrete protective wall on explosion shock wave," Journal of Vibration and Shock, vol. 32, no. 24, pp. 192-197, 2013.

[3] G. K. Schleyer, M. J. Lowak, M. A. Polcyn, and G. S. Langdon, "Experimental investigation of blast wall panels under shock pressure loading," International Journal of Impact Engineering, vol. 34, no. 6, pp. 1095-1118, 2006.

[4] C. Mu and G. Wang, "Study on numerical simulation of explosive shock waves rounding walls," Engineering Blasting, vol. 14, no. 2, pp. 16-19, 2008

[5] X. Shao and Y. Zhao, "The diffraction law of explosive shock waves acting on portable explosion-proof walls," Explosive Materials, vol. 46, no. 6, pp. 6-10, 2017.

[6] Y. Wu and Z. Song, "Research on diffraction flow effect of explosion shock wave behind blast wall," Journal of Hebei University of Engineering (Natural Science Edition), vol. 31, no. 2, pp. 9-14, 2014.

[7] T. D. Ngo, "Blast loading and blast effects on structures-an overview," Electronic Journal of Structural Engineering, vol. 7, pp. 76-91, 2007.

[8] C. Wu and H. Hao, "Modeling of simultaneous ground shock and airblast pressure on nearby structures from surface explosions," International Journal of Impact Engineering, vol. 31, no. 6, pp. 699-717, 2005.

[9] L. Chen, Z. Li, and R. Chen, "Study on the characteristics of shock wave of explosive charge," Explosion and Shock Waves, vol. 40, pp. 1-10, 2019.

[10] Z. Zhang, Z. Zhang, M. Li, T. Ge, and T. Qian, "Numerical simulation of wave dissipating performance of rapid assembling anti-blast wall," Blasting, vol. 34, no. 3, pp. 157-216, 2017.

[11] X. Nian, Y. Zhang, C. Sun, H. Wang, and D. Yan, "Analysis of transmission and diffraction effects of air shock wave upon flexible explosion-proof walls," Engineering Mechanics, vol. 32, no. 3, pp. 241-248+256, 2015.

[12] Y.-l. Ma, L.-j. Zhao, and J.-x. Nie, "Numerical Simulation on different blast walls resisting air shock wave," Blasting, vol. 27, no. 1, pp. 26-30, 2010.

[13] R. Zong and P. Bai, "Design and numerical simulation of new fence blast wall,” Blasting, vol. 33, no. 3, pp. 140-145, 2016. 
[14] W.-j. Yu, J.-c. Zhao, J.-y. Shi, and J.-h. Gong, "Study on dynamic mechanical preformance of corrugated sheet blast wall under blast loading," Sichuan Building Science, vol. 38, no. 2, pp. 78-81, 2012.

[15] J. Shi, Y. Zhu, G. Chen, J. Fu, and S. Liu, "Assessment of blast resistance capacities of corrugated blast walls based on P-I Model," Journal of Vibration and Shock, vol. 36, no. 6, pp. 188-195, 2017.

[16] R. Yang, J. Zuo, and G. Yang, "A experimental study on slotted cartridge directional controlled blasting," Journal of Vibration and Shock, vol. 37, no. 24, pp. 24-29, 2018.

[17] L. Zhang, Performance of Blasting Equipment and Explosion Effect Test, China University of Science and Technology Press, Anhui, China, 2006. 